\title{
The Orbital Motion of a Steel Ball Around a Magnetic Pole.
}

BY R. W. IVOOD.

T N Volume IV., No. 5 of the Physical Review I described an experiment for illustrating orbital motion by projecting a steel bicycle ball over a glass plate, perforated by the pole-piece of a large electro-magnet.

Several criticisms appeared in Nature and elsewhere, claiming that, under the conditions, the attractive force would vary not as the inverse square, but as the inverse fifth power of the distance. The authors of these notes appear to have overlooked the fact that the ball does not move in along a line of force, being urged towards the center by the horizontal component only, the vertical producing pressure on the plate. If the plate is over the magnetic pole this component becomes zero at the center instead of $\infty$, which would of course be the value in an hypothetical case only.

With a view of determining whether some position of the plate could be found, giving to the horizontal component a law approximating the law of gravitation within a certain area, I suggested to one of our students an experimental study of the attractive force between the magnet and the ball moving in on different planes. These experiments Professor Austin has already alluded to in a letter recently published in Nature, but it seems worth while to give the results a little more in detail, together with their bearing on the experiment.

The first experiments were made with the magnet in a horizontal position, and the ball suspended against a vertical glass plate, from a pan of a balance placed high above the magnet. This method proved unsatisfactory owing to the friction caused by the pressure of the ball against the plate.

Very good results were obtained, however, by fastening the ball to a

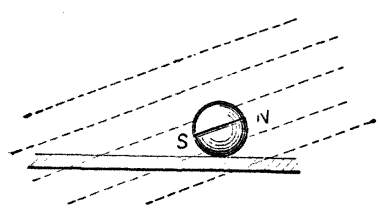
strip of tempered brass, and measuring the deflections by means of a mirror and scale. A large number of series of observations were made of which two are given below.

The pole of the magnet was at some little distance within the end of the bar. The first series shows the variation in the attractive force when the ball moved in on a plane $3 \mathrm{cms}$. above the pole. $x$ is the distance from 
the axis of the magnet, $y$ the deflection which is proportional to the attractive force.

$\begin{array}{rc}\text { Distance of Plane from Pole, } 3 \mathrm{cms} . \\ x & y \\ 4 & 2.10 \\ 5 & 1.37 \\ 6 & .91 \\ 7 & .69 \\ 8 & .51 \\ 9 & .40 \\ 10 & .31 \\ 12 & .20 \\ 14 & .12\end{array}$

Plane passing through Pole.
$x$

Calculating the attractive force for each of these two conditions from the formula $y=\frac{c}{x^{a}}$ when $c$ and $a$ are unknown constants we find that for the first series $a$ has a value of about 2. I indicating an attractive force earing very nearly as the inverse square. For the second series, where the plane in which the ball moves passes through the pole, the value of $a$ is about 3 , the force being as the inverse cube. In both cases the law of attraction changes from point to point, which is what one would expect, but within the region in which the orbits are uusally contained, it is approximately that of the inverse square or cube depending on the position of the plane.

These results indicate that planetary motion would be better represented on a plate a little above the pole piece, and this I found to be the case. With the plate at a distance of $3 \mathrm{cms}$. above the pole I have succeeded in making the ball make two revolutions in an elliptic orbit. I have abandoned the smoked plate as the friction is greater, and the numerous failures are very exasperating when each records itself, and practically spoils the appearance of the plate. The motion of the ball can be seen from a considerable distance and the experiment is on the whole more striking. I find that a grooved inclined plane is the best device for starting the ball, as its direction can be easily adjusted and the velocity varied at will. It is a good plan to draw the temper of the steel by heating the ball red hot over a Bunsen burner and cooling it slowly. On projecting the ball with moderate velocity along a line passing some $20 \mathrm{cms}$. from the pole I find that it will often complete five or six complete revolutions in a very large orbit, moving slower and slower and eventually coming to rest without "falling into the sun." This is a very striking experiment, as it is of six or eight seconds' duration, but it is best to stop the ball before it comes to rest, if one wishes to palm it off as planetary motion! It is, of course, not a case of true orbital motion, 
though none the less interesting. It is due to the permanent magnetization of the ball, the directive force of the field preventing it from rolling either towards or away from the pole. The ball sets itself with its magnetic axis along the lines of force, which make an angle with the plate, as shown in the figure, and if it rolls a little either to the left or right the directive force will roll it back. This can be shown by pushing it a trifle either towards or away from the center after it has come to rest. In both cases it rolls back at once to its original position. It has been practically rolling in a magnetic groove, if one may use the term, the attractive force playing no part. The temper of the steel should not be drawn for this experimens.

Physical Laboratory of the University of Wisconsin, Madisor, June, 1898 . 